\title{
Prethermalization in a classical phonon field: Slow relaxation of the number of phonons
}

\author{
François Huveneers $\circledast^{1, *}$ and Jani Lukkarinen $\circledast^{2, \dagger}$ \\ ${ }^{1}$ Université Paris Dauphine-PSL, CEREMADE, Place du Maréchal de Tassigny, 75016 Paris, France \\ ${ }^{2}$ Department of Mathematics and Statistics, University of Helsinki, P.O. Box 68, FI-00014 Helsingin yliopisto, Finland
}

(Received 3 March 2020; revised manuscript received 9 April 2020; accepted 10 April 2020; published 8 May 2020)

\begin{abstract}
We investigate the emergence of an astonishingly long prethermal plateau in a classical phonon field, here, a harmonic chain with on-site pinning. Integrability is broken by a weak anharmonic on-site potential with strength $\lambda$. In the small $\lambda$ limit, the approach to equilibrium of a translation invariant initial state is described by kinetic theory. However, when the phonon band becomes narrow, we find that the (nonconserved) number of phonons relaxes on much longer timescales than kinetic. We establish rigorous bounds on the relaxation time and develop a theory that yields exact predictions for the dissipation rate in the limit $\lambda \rightarrow 0$. We compare the theoretical predictions with data from molecular-dynamics simulations and find good agreement. Our Rapid Communication shows how classical systems may exhibit phenomena which, at the first glance, appear to require quantization.
\end{abstract}

DOI: 10.1103/PhysRevResearch.2.022034

I. Introduction. Thermalization is one of the most commonly encountered physical phenomena, and yet, it still remains poorly understood. Several materials have been found where the approach to equilibrium can be drastically slowed down or even suppressed: Anderson insulators [1], many-body localized chains [2,3], ergodic systems featuring many-body scars [4], quantum glasses [5], Fermi-Pasta-UlamTsingou chains [6], classical nonlinear disordered lattices [7], etc. Moreover, some systems with otherwise good ergodic properties may feature extensive pseudoconserved quantities that relax only on very long timescales [8-23]. The period during which this quantity stays approximately conserved provides an example of a prethermal state which can host an abundance of fascinating physical phenomena [24-28].

In this Rapid Communication, we investigate a classical many-body Hamiltonian $H=H_{0}+\lambda V$ in the limit $\lambda \rightarrow 0$, where $H_{0}$ is integrable (a harmonic lattice or free phonon field), and $V$ breaks integrability (an on-site anharmonic potential). See Sec. II for a precise definition. If the system is started in a translation invariant state, its state evolves swiftly to the generalized Gibbs ensemble (GGE) characterized by all the conserved quantities of $H_{0}$ [29-32]. Next, according to kinetic theory and the Boltzmann-Peierls equation, it approaches equilibrium in a time $\tau_{1}$ with $\tau_{1} \sim \lambda^{-2}$ for $\lambda \rightarrow 0$ [33-36]. However, if the phonon band is sufficiently narrow, the (nonconserved) number of phonons is preserved by kinetic processes $[35,37]$, and only a prethermal plateau is reached

\footnotetext{
*huveneers@ceremade.dauphine.fr

†jani.lukkarinen@helsinki.fi
}

Published by the American Physical Society under the terms of the Creative Commons Attribution 4.0 International license. Further distribution of this work must maintain attribution to the author(s) and the published article's title, journal citation, and DOI. on kinetic timescales. As our analysis reveals, the proper equilibrium is only reached after a longer time $\tau_{2}$, scaling as $\tau_{2} \sim \lambda^{-2 p}$ for some $p \geqslant 1$ which becomes arbitrarily large as the width of the band narrows to zero. See Fig. 1 for a summary of the above process.

The presence of an almost conserved quantity (or adiabatic invariant) for the Hamiltonian $H$ studied in this Rapid Communication has been realized in Refs. [19,20]. The slow dissipation of some quantized fields $[13,15]$ is a very much related phenomenon, even though that seemed, at first sight, to require quantization. Here, we develop an analogy with a quantum model which serves as a stepping stone to understanding the slow dissipation of the number of phonons, and we state rigorous results further substantiating the connection, see Sec. III. Next, we provide a theory to compute the dissipation rate of the pseudoconserved quantity, which we are able to back with numerical results, see Sec. IV. General predictions for the rate have been derived in Ref. [38] for quantum systems, see also Refs. [39-41]. However, we note that our system is classical and that an extra timescale is present since relaxation to the prethermal plateau involves kinetic processes.

II. Model. Let the Hamiltonian $H$ be given by

$H=\sum_{x \in \mathbb{Z}} \frac{p_{x}^{2}}{2}+\frac{\omega_{0}^{2}}{2} q_{x}^{2}-\frac{\omega_{0}^{2} \boldsymbol{\delta}}{2}\left(q_{x-1} q_{x}+q_{x} q_{x+1}\right)+\frac{\lambda}{r} q_{x}^{r}$,

with $r>2$ as an even integer (below, we focus on $r=4,6$ ) and $\omega_{0}>0$ as a characteristic frequency of the system. The parameter $\delta$ determines the width of the phonon band, and we have highlighted it here to facilitate following its role in the following results and computations. The dynamics is classical and governed by Hamilton's equations: $\dot{q}_{x}=p_{x}$ and

$$
\dot{p}_{x}=-(1-2 \delta) \omega_{0}^{2} q_{x}+\delta \omega_{0}^{2}\left(q_{x+1}-2 q_{x}+q_{x}\right)-\lambda q_{x}^{r-1},
$$

where $\dot{f}$ denotes the time derivative of $f$. Stability imposes $\lambda \geqslant 0$ and $0 \leqslant \delta \leqslant 0.5$. The chain is uncoupled for $\delta=0$ and 


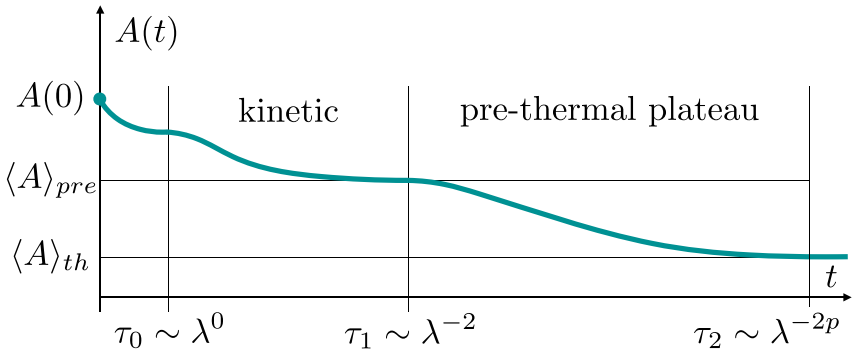

FIG. 1. Expected time evolution of a local observable $A(t)$.

unpinned for $\delta=0.5$, and we restrict our attention to $0<\delta<$ 0.5 . If needed, we may obviously restrict the summation in Eq. (1) to $1 \leqslant x \leqslant L$ for a length $L$ and consider the limit $L \rightarrow \infty$ only afterwards.

For $\lambda=0$, the chain is harmonic. For a pseudomomentum $k$ in the Brillouin zone $\mathrm{BZ}=]-0.5,0.5]$, let the two related phonon modes $a^{ \pm}(k)$ be defined by

$$
a^{ \pm}(k)=\frac{1}{\sqrt{2}}\left(\omega^{1 / 2}(k) \hat{q}(k) \mp i \frac{1}{\omega^{1 / 2}(k)} \hat{p}(k)\right),
$$

where $\omega$ denotes the dispersion relation,

$$
\omega(k)=\omega_{0}[1-2 \delta \cos (2 \pi k)]^{1 / 2},
$$

and $\hat{f}$ denotes the Fourier transform of $f$, defined by $\hat{f}(k)=$ $\sum_{x \in \mathbb{Z}} f(x) e^{-2 i \pi k x}$. We identify $n(k)=|a(k)|^{2}$ with the number of phonons with pseudomomentum $k$, and we denote the total number of phonons by $N_{0}=\int_{\mathrm{BZ}} d k n(k)$. From the analyticity of $\omega(k)$ in Eq. (3), we deduce that $N_{0}$ is quasilocal, i.e.,

$N_{0}=\sum_{x, y} K_{q q}(x-y) q_{x} q_{y}+K_{q p}(x-y) q_{x} p_{y}+K_{p p}(x-y) p_{x} p_{y}$,

where the kernels $K_{\star}(z)$ decay exponentially with $|z|$.

When $\lambda>0$, the Hamiltonian (1) can be written as $H=$ $H_{0}+\lambda V$ with

$$
H_{0}=\int_{\mathrm{BZ}} d k \omega(k) n(k) \text { and }
$$

$V=\frac{1}{r 2^{r / 2}} \int_{(\mathrm{BZ})^{r}} d k_{1} \cdots d k_{r} \frac{\delta\left(k_{1}+\cdots+k_{r}\right)}{\left(\omega_{1} \cdots \omega_{r}\right)^{1 / 2}} \sum_{\sigma_{j}= \pm} a_{1}^{\sigma_{1}} \cdots a_{r}^{\sigma_{r}}$,

with the notations $\omega_{j}=\omega\left(k_{j}\right)$ and $a_{j}^{\sigma_{j}}=a^{\sigma_{j}}\left(k_{j}\right)$.

The evolution of an observable $A$, i.e., a function $A$ on the phase-space $\mathbb{R}^{2 L}$, is given by $\dot{A}=\{A, H\}$, where $\{\cdot, \cdot\}$ denotes the usual Poisson bracket. The observables $a^{ \pm}(k)$ are a classical analog of creation/annihilation operators for bosons with pseudomomentum $k$ as evidenced by the Poisson bracket rule $\left\{a_{k}^{\sigma}, a_{k^{\prime}}^{\sigma^{\prime}}\right\}=i \sigma \delta_{\sigma+\sigma^{\prime}} \delta\left(k+k^{\prime}\right)$. From this and from Eq. (4), we deduce that $N_{0}$ is not conserved, i.e., $\left\{H, N_{0}\right\} \neq 0$ due to the terms with $\sum_{j=1}^{r} \sigma_{j} \neq 0$ in Eq. (4).

III. Pseudoconservation of $N_{0}$. To compute the timescales on which $N_{0}$ gets dissipated, let us first assume that the system is quantized, i.e., that $a^{ \pm}$'s are creation/annihilation operators for bosons. Later on, we will see how the conclusions carry over to the classical system. In this case, $N_{0}$ has an integer spectrum. In the limit $\lambda \rightarrow 0$, only resonant processes, preserving the bare energy $H_{0}$, do effectively destroy the

\begin{tabular}{|c|c|c|}
\hline & $r=4$ & $r=6$ \\
\hline $0.3<\delta<0.5$ & $p=2$ & $p=1$ \\
\hline $0.25<\delta<0.3$ & $p=3$ & $p=2$ \\
\hline$\delta \rightarrow 0$ & $p \sim 1 / \delta$ & $p \sim 1 / 2 \delta$ \\
\hline
\end{tabular}

TABLE I. Order of the processes $\left(\lambda^{p}\right)$ destroying effectively the conservation of $N_{0}$ for $r=4,6$ in Eq. (4).

conservation of $N_{0}$. Therefore, in first order in $\lambda$, the process of creating two phonons (since $r$ is even, it is not possible to create only one phonon) must satisfy the constraints,

$$
\begin{aligned}
\omega_{1}+\cdots+\omega_{n / 2+1}-\omega_{n / 2+2}-\cdots-\omega_{n} & =0, \\
k_{1}+\cdots+k_{n} & =0,
\end{aligned}
$$

where $n=r$ (we will later consider $n \neq r$ when dealing with higher-order processes), and where the second constraint is taken mod 1 and stems from translation invariance, cf. Eq. (4). Since the width of the dispersion relation in (3) scales as $2 \omega_{0} \delta$ for small $\delta$, larger and larger values of $r$ are needed to satisfy the constraints in Eq. (5). Thus, for given $n \geqslant 2$ even, there exists $\boldsymbol{\delta}_{c}(n)$ such that Eq. (5) only has solutions for $\delta \geqslant \delta_{c}(n): \delta_{c}(2)=\delta_{c}(4)=0.5$ (exact), $\delta_{c}(6)=0.3$ (exact), $\delta_{c}(8) \simeq 0.25$ (numerical), and, in general, $\delta_{c}(n) \sim 2 / n$ for $n \rightarrow \infty$, see the Supplemental Material (SM) [42]. From now on, we will assume that $\boldsymbol{\delta}$ is such that $\boldsymbol{\delta} \neq$ $\boldsymbol{\delta}_{c}(n)$ for any $n$, leaving these exceptional cases for further studies.

Higher-order processes need obviously to be taken into account in the case of $\delta<\delta_{c}(r)$. The analysis detailed in the SM [42] yields that a process of order $\lambda^{p}$ involves the creation/annihilation of $n=p(r-2)+2$ phonons, and the above analysis carries over with this new value for $n$. The value $n=p(r-2)+2$ may also be recovered from a simple power counting argument: Let $\operatorname{ad}_{H}=\{H, \cdot\}(=-i[H, \cdot]$ for a quantum system with $\hbar=1)$ and expand $e^{-\operatorname{tad}_{H}} N_{0}$ at order $p$; this yields $(-t)^{p} \operatorname{ad}_{H}^{p}\left(N_{0}\right) / p$ ! which is a polynomial of order $n$ in $a^{ \pm}$with $n=p(r-2)+2$ as above. Given $r$ and $\delta$, we may now determine the smallest $p$ so that a process of order $\lambda^{p}$ destroys $N_{0}$ effectively: $p \geqslant 1$ is the unique integer for which

$$
\boldsymbol{\delta}_{c}[p(r-2)+2]<\delta<\boldsymbol{\delta}_{c}[(p-1)(r-2)+2] .
$$

Explicit results are gathered in Table I.

We observe that, even though quantitative statements are obviously model dependent, and, in particular, the threshold values $\boldsymbol{\delta}_{c}$ depend on the specific form of the dispersion relation $\omega(k)$ in Eq. (3), the conclusion that $p \sim c / \delta$ as $\delta \rightarrow 0$ is generic (for a polynomial interaction $V$ ), following from the fact that the width of the band in $\omega(k)$ decreases to zero as $\delta \rightarrow 0$.

The above analysis may be turned into rigorous results using the formalism developed in Ref. [13] that can be straightforwardly adapted to a classical system through the canonical replacement of $-i[H, \cdot]$ by $\{H, \cdot\}$, see the SM [42] and below. The key observation to proceed is that, even though $N_{0}$ is no longer quantized, the spectrum of $\operatorname{ad}_{N_{0}}=\left\{N_{0}, \cdot\right\}$ is as 
follows:

$$
\left\{N_{0}, a_{1}^{\sigma_{1}} \cdots a_{m}^{\sigma_{m}}\right\}=i\left(\sigma_{1}+\cdots+\sigma_{m}\right) a_{1}^{\sigma_{1}} \cdots a_{m}^{\sigma_{m}} .
$$

Hence, it acts formally in the same way as the quantum superoperator $-i\left[N_{0}, \cdot\right]$, and this is eventually what matters.

Let us fix $r$ and $\boldsymbol{\delta}$ such that $p>1$, and let us first express a result in a formulation directly inspired from Ref. [13]: There exists a canonical change in variables, bringing $H$ into $\tilde{H}$ so that $\left\{\tilde{H}, N_{0}\right\}=O\left(\lambda^{p}\right)$ where the term $O\left(\lambda^{p}\right)$ is extensive. To formulate a precise claim, we will consider extensive observables $\varphi_{m}$ of the form

$$
\begin{aligned}
\varphi_{m}= & \int_{(\mathrm{BZ})^{m}} d k_{1} \cdots d k_{m} \delta\left(k_{1}+\cdots+k_{m}\right) \\
& \times \sum_{\sigma_{j}= \pm} \hat{\varphi}_{m}\left(k_{1}, \ldots, k_{m}, \sigma_{1}, \ldots, \sigma_{m}\right) a_{1}^{\sigma_{1}} \cdots a_{m}^{\sigma_{m}},
\end{aligned}
$$

where $m \geqslant 2$ and where $\hat{\varphi}_{m}$ is analytic in $\left(k_{1}, \ldots, k_{m}\right)$, ensuring that $\varphi_{m}$ is a sum of quasilocal observables. A function $\varphi$ will simply be said to be a polynomial (of order $m$ ) if it is of the form $\varphi=\sum_{k=2}^{m} \varphi_{k}$ with $\varphi_{k}$ as in Eq. (7). The following claim is shown in the SM [42]:

Claim 1. Let $L$ be finite, and let us assume periodic boundary conditions. For $|\lambda|$ small enough, there exists a polynomial $G=\lambda \sum_{n=1}^{p-1} \lambda^{n-1} G_{n}$ such that $\tilde{H}:=e^{-\{G,\}} H$ is a welldefined real analytic function in a neighborhood of the origin in $\mathbb{R}^{2 L}$ and such that $\left\{\tilde{H}, N_{0}\right\}=\lambda^{p} \sum_{n=p}^{\infty} \lambda^{n-p} \mathcal{J}_{n}$, where $\mathcal{J}_{n}$ 's are polynomials and where the expansion converges to an analytic function in a neighborhood of the origin in $\mathbb{R}^{2 L}$.

Claim 1 provides a good way to think about the phenomenon but does not yield as such a very powerful result in the thermodynamic limit: The radius of convergence of $\lambda$ may shrink as $L \rightarrow \infty$ (even though we are interested in the regime $\lambda \rightarrow 0$, the limit $L \rightarrow \infty$ needs to be taken before the limit $\lambda \rightarrow 0$ ). As a way out, we may undo the above transformation to obtain a dressed number of phonons $e^{\{G, \cdot\}} N_{0}$ and then truncate its expansion at order $p-1$. Doing so, we end up with a well-defined pseudoconserved quantity $N$ in the thermodynamic limit, see the SM [42] for details:

Claim 2. Let now the chain be defined on the full lattice $\mathbb{Z}$. There exists a quantity $N=N_{0}+\lambda \sum_{n=1}^{p-1} \lambda^{n-1} N_{n}$, where $N_{n}$ are polynomials of order $n r-2(n-1)$ for $1 \leqslant n \leqslant p-1$ such that $\{H, N\}=\lambda^{p}\left\{V, N_{p-1}\right\}$.

These claims furnish upper bounds on the dissipation rate of $N$, but the determination of this rate requires the knowledge of the instantaneous state of the system. To proceed further, we will invoke additional assumptions and leave mathematical rigor behind.

IV. Evaluation of the decay rate. For $p=1, N=N_{0}$. The dissipation rate $\gamma$ of the density $N_{0} / L$ in an instantaneous state $\rho$ and in the infinite volume limit is given by $\gamma=\langle J\rangle_{\rho} / \delta(0)$ with a flux $J=\lambda \mathcal{J}=\lambda\left\{V, N_{0}\right\}$ and where $\delta(0)$ stands for the infinite volume, corresponding to $L$ in a chain of finite length. If the system is prepared in a translation invariant state with zero average, after a short transient time, it evolves towards a GGE characterized by a Wigner function $W$, i.e., a Gaussian state $\exp \left[-\int_{\mathrm{BZ}} d k n(k) / W(k)\right] / Z$. Usual kinetic theory yields the following expression for the rate $\gamma(W)$ : Given a function

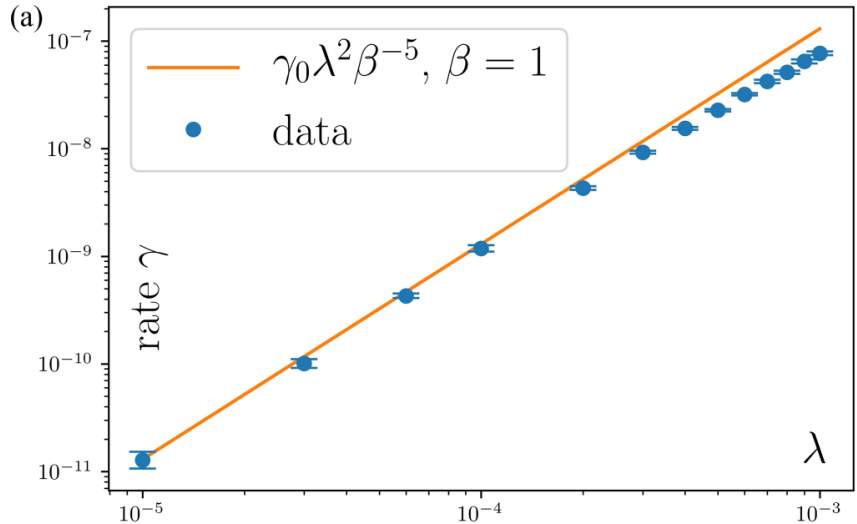

(b)

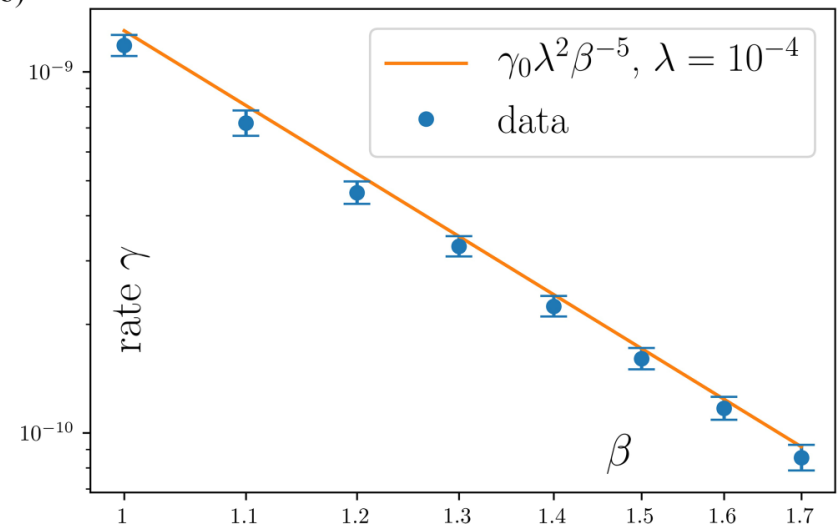

FIG. 2. $\gamma$ as a function of (a) $\lambda$ and (b) $\beta$ for $r=6$ and $\delta=0.35$. Kinetic theory, i.e., Eq. (8), predicts $\gamma=\gamma_{0} \lambda^{2} \beta^{-5}$ with $\gamma_{0} \simeq 0.13$ for $\lambda \rightarrow 0$.

$\varphi=\int_{\mathrm{BZ}} d k \hat{\varphi}(k) n(k)$, let $J_{\varphi}=\lambda \mathcal{J}_{\varphi}=\{H, \varphi\}=\lambda\{V, \varphi\}$, then

$\gamma(W)=\frac{\lambda^{2}}{\delta(0)} \lim _{\tau \rightarrow \infty} \int_{0}^{\infty} d t e^{-t / \tau}\left\langle\mathcal{J}_{1}(0) \mathcal{J}_{1 / W}(t)\right\rangle_{W}+O\left(\lambda^{3}\right)$,

where $\langle\cdot\rangle_{W}$ denotes the average over the GGE and where the dynamics in the time integral is the free dynamics $(\lambda=0)$. Expression (8) can, thus, be evaluated explicitly in leading order. In the SM [42], we provide a derivation of Eq. (8) which is fully consistent with the derivation used in the more general case of $p>1$. We tested numerically the validity of Eq. (8) for $r=6$ and $\delta=0.35$ for an out-of-equilibrium initial state corresponding to $W=[\beta(\omega(k)-\mu)]^{-1}$ with $\mu=-1$ and various values of $\beta$. We found excellent agreement with the value of $\gamma(W)$ extracted from direct simulation of the dynamics, see Fig. 2. See the SM [42] for the numerical protocol.

For $p>1$, we find it convenient to move to the rotated frame where $N_{0}$ is the pseudoconserved quantity of $\tilde{H}$, see Claim 1. The GGE is now parametrized by only two extensive quantities $\tilde{H}$ and $N_{0}$. Our main assumption (to be discussed later on) is that the system is in a state $\rho, \lambda^{p}$ close to the GGE $\rho_{0}$,

$$
\rho=\rho_{0}\left[1+\lambda^{p} f+O\left(\lambda^{p+1}\right)\right], \quad \rho_{0} \sim e^{-\beta\left(\tilde{H}-\mu N_{0}\right)},
$$


(a)

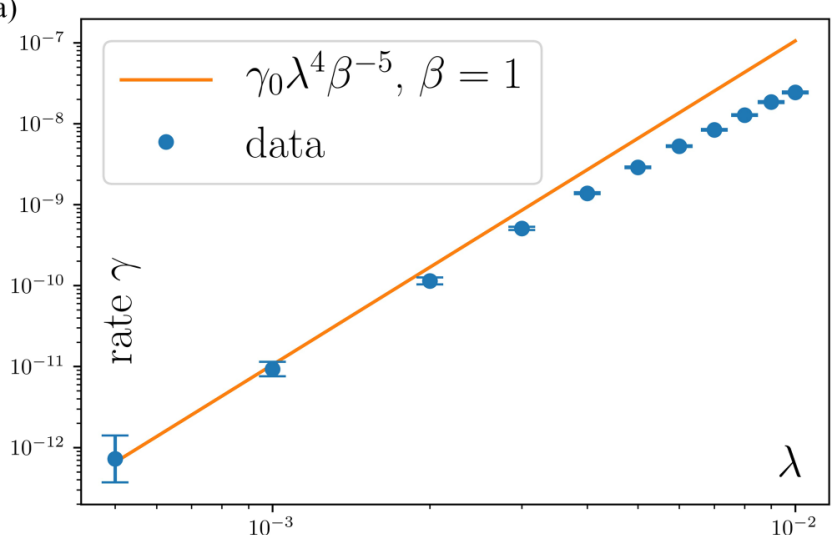

(b)

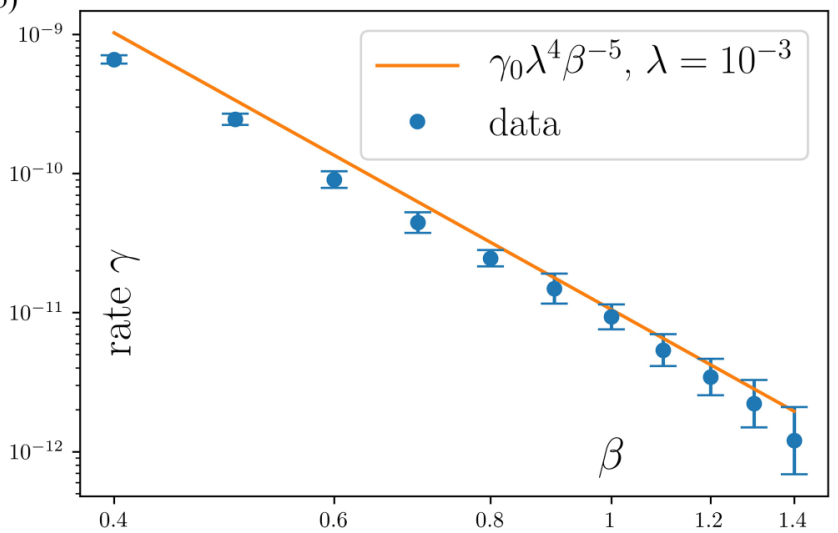

FIG. 3. $\gamma$ as a function of (a) $\lambda$ and (b) $\beta$ for $r=4$ and $\delta=0.45$. Our theory, Eq. (10), predicts $\gamma=\gamma_{0} \lambda^{4} \beta^{-5}$ with $\gamma_{0} \simeq 10.5$.

where $f$ is some correction that will be determined by maximizing local stationarity. The key observation is that $\langle J\rangle_{\rho_{0}}=0$ where $J:=\lambda^{p} \mathcal{J}=\left\{\tilde{H}, N_{0}\right\}$ [38]. Indeed,

$$
e^{-\beta\left(\tilde{H}-\mu N_{0}\right)}\left\{\tilde{H}, N_{0}\right\}=-\frac{1}{\beta}\left\{e^{-\beta\left(\tilde{H}-\mu N_{0}\right)}, N_{0}\right\},
$$

and the integral of a Poisson bracket vanishes. Hence, the instantaneous dissipation rate $\gamma(\beta, \mu)$ may be written as

$$
\gamma(\beta, \mu)=\langle J\rangle_{\rho}=\lambda^{2 p}\langle\mathcal{J} f\rangle_{\rho_{0}}+O\left(\lambda^{2 p+1}\right) .
$$

Since this rate scales as $\lambda^{2 p}$, the state $\rho$ itself evolves on that timescale. Stationarity on shorter timescales determines $f$, and an explicit computation yields, see the SM [42],

$$
\begin{aligned}
\gamma(\beta, \mu)= & \frac{\beta \mu \lambda^{2 p}}{\delta(0)} \lim _{\tau \rightarrow \infty} \int_{0}^{\infty} d t e^{-t / \tau}\langle\mathcal{J}(0) \mathcal{J}(t)\rangle_{\rho_{0}} \\
& +O\left(\lambda^{2 p+1}\right) .
\end{aligned}
$$

Again, the dynamics in the time integral is the dynamics generated by $H_{0}$, and $\gamma(\beta, \mu)$ can, thus, be computed explicitly in leading order.

We performed two sets of tests all of which have $p=2$ (accessing larger values of $p$ would require too long simulation times). We start from a state of the type $\rho_{0}$ with $\mu=-1$ and various values of $\beta$. For $r=4$ and $\delta=0.45$, the results reported in Fig. 3 show very good agreement between the prediction from Eq. (10) and direct simulation of the dynamics. For $r=6$ and $\boldsymbol{\delta}=0.28$, the observed rate is
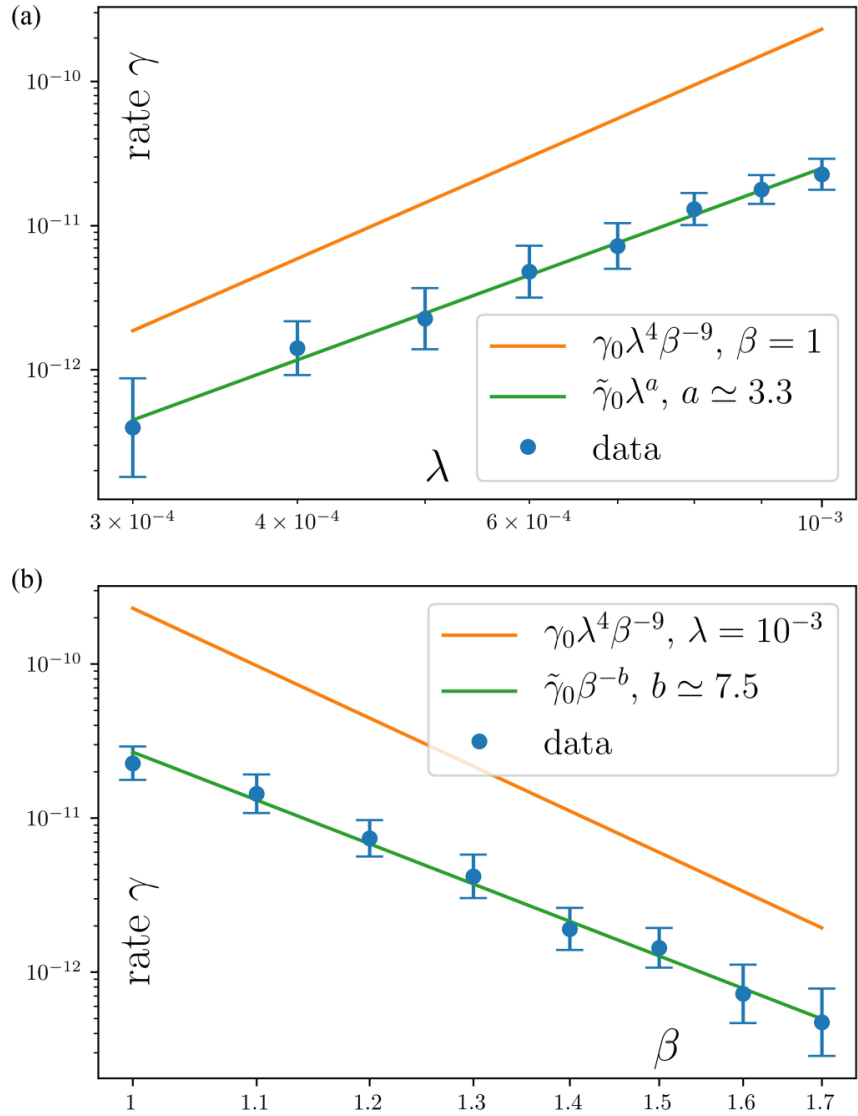

FIG. 4. $\gamma$ as a function of (a) $\lambda$ and (b) $\beta$ for $r=6$ and $\delta=0.28$. Our theory, Eq. (10), predicts $\gamma=\gamma_{0} \lambda^{4} \beta^{-9}$ with $\gamma_{0} \simeq 230$.

significantly smaller than the one predicted by Eq. (10), but it decreases slower as a function of $\lambda$ and $\beta^{-1}$, see Fig. 4. Comparing with the discrepancies at the largest values of $\lambda$ on the upper panel of Fig. 3, makes it plausible that our theory just needs smaller values of $\lambda$ to be validated. Besides, the fact that the observations are below the theoretical predictions is a second indication that the theory will become accurate for smaller values of $\lambda$ since a smaller rate guarantees that our main hypothesis, Eq. (9), from which our predictions follow, is more easily satisfied.

Irrespective of numerical observations, we finally would like to perform a consistency check of the main assumption in Eq. (9). On one hand, due to the dissipation of $N_{0}$, the state $\rho_{0}$ evolves with time at a rate of $v_{1} \sim \lambda^{2 p}$, i.e., $\beta, \mu$ evolve at this rate in order to yield correct values for $\langle\tilde{H}\rangle_{\rho_{0}}$ and $\left\langle N_{0}\right\rangle_{\rho_{0}}$. On the other hand, the system relaxes toward the instantaneous pseudoequilibrium $\rho_{0}$ through kinetic processes. Assuming that state $\rho$ is at a distance of order $\lambda^{p}$ from $\rho_{0}$ as required by Eq. (9), we conclude that it moves at a rate of $v_{2} \sim \lambda^{2} \lambda^{p}$. Consistency of the theory requires that $v_{1} \lesssim v_{2}$, i.e., that the instantaneous fixed point $\rho_{0}$ moves slow enough so that state $\rho$ has the time to relax to it. Clearly, this is wrong for $p=1$, marginal for $p=2$, and fine for $p>2$. We had treated separately the case of $p=1$ since, indeed, there is no reason to think that the prethermal state should be characterized by the two parameters $\beta, \mu$ only. Unfortunately, the above argument is not conclusive for $p=2$, whereas numerical data are only available in this case. 
V. Conclusions and outlook. Our Rapid Communication provides an example of a long prethermal plateau; it shows how a phenomenology initially explored in quantum systems carries over to a classical setup, and it participates in recent efforts to describe accurately the dissipation of pseudoconserved quantities. The main features of our theory carry over to $d>1$ and, for $d=3$, we may contemplate the possibility of realizing a prethermal Bose-Einstein condensate in this classical system, exploiting the conservation of the number of phonons over a very long period.

Acknowledgments. We thank W. De Roeck and H. Spohn for helpful discussions and $\mathrm{C}$. Mendl for providing the original code for numerical simulations. F.H. and J.L. benefited from the support of the Project No. EDNHS ANR-14CE25-0011, and F.H. benefited from Project No. LSD ANR15-CE40-0020-01 of the French National Research Agency (ANR) as well as from the support of the International Centre for Theoretical Sciences (ICTS) during a visit for the program-Thermalization, Many body localization and Hydrodynamics (Code No. ICTS/hydrodynamics2019/11). The work has also been supported by the Academy of Finland via the Centre of Excellence in Analysis and Dynamics Research (Project No. 307333) and the Matter and Materials Profi4 university profiling action.
[1] P. W. Anderson, Absence of diffusion in certain random lattices, Phys. Rev. 109, 1492 (1958).

[2] I. V. Gornyi, A. D. Mirlin, and D. G. Polyakov, Interacting Electrons in Disordered Wires: Anderson Localization and Low-T Transport, Phys. Rev. Lett. 95, 206603 (2005).

[3] D. M. Basko, I. L. Aleiner, and B. L. Altshuler, Metal-insulator transition in a weakly interacting many-electron system with localized single-particle states, Ann. Phys. (NY) 321, 1126 (2006).

[4] C. J. Turner, A. A. Michailidis, D. A. Abanin, M. Serbyn, and Z. Papic, Weak ergodicity breaking from quantum many-body scars, Nat. Phys. 14, 745 (2018).

[5] Y. Kagan and L. Maksimov, Localization in a system of interacting particles diffusing in a regular crystal, Zh. Eksp. Teor. Fiz. 87, 348 (1984)

[6] E. Fermi, P. Pasta, S. Ulam, and M. Tsingou, Studies of the nonlinear problems, Los Alamos Scientific Laboratory Technical report, 1955 (unpublished).

[7] D. Basko, Weak chaos in the disordered nonlinear Schrödinger chain: destruction of Anderson localization by Arnold diffusion, Ann. Phys. (NY) 326, 1577 (2011).

[8] L. D'Alessio and A. Polkovnikov, Many-body energy localization transition in periodically driven systems, Ann. Phys. (NY) 333, 19 (2013).

[9] L. D'Alessio and M. Rigol, Long-time Behavior of Isolated Periodically Driven Interacting Lattice Systems, Phys. Rev. X 4, 041048 (2014).

[10] A. Lazarides, A. Das, and R. Moessner, Equilibrium states of generic quantum systems subject to periodic driving, Phys. Rev. E 90, 012110 (2014)

[11] D. A. Abanin, W. De Roeck, and F. Huveneers, Exponentially Slow Heating in Periodically Driven Many-Body Systems, Phys. Rev. Lett. 115, 256803 (2015).

[12] D. A. Abanin, W. De Roeck, W. W. Ho, and F. Huveneers, Effective Hamiltonians, prethermalization, and slow energy absorption in periodically driven many-body systems, Phys. Rev. B 95, 014112 (2017)

[13] D. A. Abanin, W. De Roeck, W. W. Ho, and F. Huveneers, A rigorous theory of many-body prethermalization for periodically driven and closed quantum systems, Commun. Math. Phys. 354, 809 (2017).

[14] T. Mori, T. Kuwahara, and K. Saito, Rigorous Bound on Energy Absorption and Generic Relaxation in Periodically Driven Quantum Systems, Phys. Rev. Lett. 116, 120401 (2016).
[15] N. Strohmaier, D. Greif, R. Jördens, L. Tarruell, H. Moritz, T. Esslinger, R. Sensarma, D. Pekker, E. Altman, and E. Demler, Observation of Elastic Doublon Decay in the FermiHubbard Model, Phys. Rev. Lett. 104, 080401 (2010).

[16] R. Sensarma, D. Pekker, E. Altman, E. Demler, N. Strohmaier, D. Greif, R. Jördens, L. Tarruell, H. Moritz, and T. Esslinger, Lifetime of double occupancies in the Fermi-Hubbard model, Phys. Rev. B 82, 224302 (2010).

[17] S. Vajna, K. Klobas, T. Prosen, and A. Polkovnikov, Replica Resummation of the Baker-Campbell-Hausdorff Series, Phys. Rev. Lett. 120, 200607 (2018).

[18] W. De Roeck and V. Verreet, Very slow heating for weakly driven quantum many-body systems, arXiv:1911.01998.

[19] A. Carati and A. M. Maiocchi, Exponentially long stability times for a nonlinear lattice in the thermodynamic limit, Commun. Math. Phys. 314, 129 (2012).

[20] A. Giorgilli, S. Paleari, and T. Penati, An extensive adiabatic invariant for the Klein-Gordon model in the thermodynamic limit, Ann. Henri Poincaré 16, 897 (2015).

[21] A. Rajak, R. Citro, and E. G. Dalla Torre, Stability and prethermalization in chains of classical kicked rotors, J. Phys. A: Math. Theor. 51, 465001 (2018).

[22] A. Rajak, I. Dana, and E. G. Dalla Torre, Characterizations of prethermal states in periodically driven many-body systems with unbounded chaotic diffusion, Phys. Rev. B 100, 100302(R) (2019).

[23] O. Howell, P. Weinberg, D. Sels, A. Polkovnikov, and M. Bukov, Asymptotic Prethermalization in Periodically Driven Classical Spin Chains, Phys. Rev. Lett. 122, 010602 (2019).

[24] D. V. Else, B. Bauer, and C. Nayak, Prethermal Phases of Matter Protected by Time-Translation Symmetry, Phys. Rev. X 7, 011026 (2017).

[25] D. V. Else, W. W Ho, and P. T. Dumitrescu, Long-lived interacting phases of matter protected by multiple time-translation symmetries in quasiperiodically-driven systems, arXiv:1910.03584.

[26] D. V. Else, P. Fendley, J. Kemp, and C. Nayak, Prethermal Strong Zero Modes and Topological Qubits, Phys. Rev. X 7, 041062 (2017).

[27] N. H. Lindner, E. Berg, and M. S. Rudner, Universal Chiral Quasisteady States in Periodically Driven Many-Body Systems, Phys. Rev. X 7, 011018 (2017).

[28] I. Martin, G. Refael, and B. Halperin, Topological Frequency Conversion in Strongly Driven Quantum Systems, Phys. Rev. X 7, 041008 (2017). 
[29] T. V. Dudnikova, A. I. Komech, and H. Spohn, On the convergence to statistical equilibrium for harmonic crystals, J. Math. Phys. 44, 2596 (2003).

[30] M. Rigol, V. Dunjko, V. Yurovsky, and M. Olshanii, Relaxation in a Completely Integrable Many-Body Quantum System: An Ab Initio Study of the Dynamics of the Highly Excited States of 1D Lattice Hard-Core Bosons, Phys. Rev. Lett. 98, 050405 (2007).

[31] L. Vidmar and M. Rigol, Generalized Gibbs ensemble in integrable lattice models, J. Stat. Mech.: Theory Exp. (2016) 064007.

[32] F. H. L. Essler and M. Fagotti, Quench dynamics and relaxation in isolated integrable quantum spin chains, J. Stat. Mech.: Theory Exp. (2016) 064002.

[33] R. Peierls, Zur kinetischen theorie der wärmeleitung in kristallen, Ann. Phys. (Berlin) 395, 1055 (1929).

[34] H. Spohn, The Phonon Boltzmann Equation, Properties and Link to Weakly Anharmonic Lattice Dynamics, J. Stat. Phys. 124, 1041 (2006).

[35] C. B. Mendl, J. Lu, and J. Lukkarinen, Thermalization of oscillator chains with onsite anharmonicity and comparison with kinetic theory, Phys. Rev. E 94, 062104 (2016).
[36] J. Lukkarinen, in Thermal Transport in Low Dimensions: From Statistical Physics to Nanoscale Heat Transfer, edited by S. Lepri (Springer International Publishing, Cham, 2016), pp. 159-214.

[37] H. Spohn, Collisional invariants for the phonon Boltzmann equation, J. Stat. Phys. 124, 1131 (2006).

[38] K. Mallayya, M. Rigol, and W. De Roeck, Prethermalization and Thermalization in Isolated Quantum Systems, Phys. Rev. X 9, 021027 (2019).

[39] Z. Lenarčič, F. Lange, and A. Rosch, Perturbative approach to weakly driven many-particle systems in the presence of approximate conservation laws, Phys. Rev. B 97, 024302 (2018).

[40] F. Lange, Z. Lenarčič, and A. Rosch, Time-dependent generalized Gibbs ensembles in open quantum systems, Phys. Rev. B 97, 165138 (2018).

[41] P. Reimann and L. Dabelow, Typicality of Prethermalization, Phys. Rev. Lett. 122, 080603 (2019).

[42] See Supplemental Material at http://link.aps.org/supplemental/ 10.1103/PhysRevResearch.2.022034 for the derivation of the estimates on $\delta_{c}$, the proofs of Claims 1 and 2, the derivation of Eqs. (8) and (10), the details of the numerical procedure, and explicit computations of the decay rate needed for the comparison between numerical and theoretical results. 